\title{
Performance analysis of demodulation with diversity - A combinatorial approach I: Symmetric function theoretical methods
}

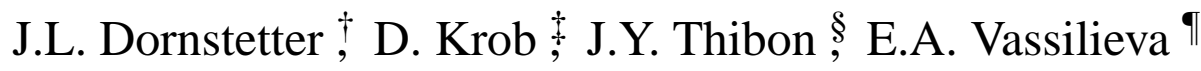 \\ received 17 Oct 2001, revised 15 Sep 2002, accepted?.
}

This paper is devoted to the presentation of a combinatorial approach, based on the theory of symmetric functions, to analyze the performance of a family of demodulation methods used in mobile telecommunications.

Keywords: Young tableau, symmetric function, Schur function, signal processing, demodulation, mobile communications

\section{Introduction}

Modulating numerical signals means transforming them into wave forms. Due to their importance in practice, modulation methods have been widely studied in signal processing (see for instance Chapter 5 of [10]). One of the most important problems in this area is the performance evaluation of the optimum receiver associated with a given modulation method, which leads to the computation of various error probabilities (see again [10]).

Among the different modulation protocols used in practice, an important class consists in methods where the modulation reference (i.e. a fixed numerical sequence) is transmitted by the same channel as the modulated signal. The demodulation decision needs then to consider at least two noisy informations, i.e. the transmitted signal and the transmitted reference. One can also take into account in the demodulating process several noisy copies of these two signals: one speaks then of demodulation with diversity. The error probabilities appearing in such contexts have the following form (cf. Section 3.1):

$$
P(U<V)=P\left(U=\sum_{i=1}^{N}\left|u_{i}\right|^{2}<V=\sum_{i=1}^{N}\left|v_{i}\right|^{2}\right),
$$

\footnotetext{
${ }^{\dagger}$ Nortel Networks - 38, rue Paul Cézanne - Guyancourt - PC MI 54 - 78928 Yvelines Cedex 9 - France - e-mail : jeanlouis.dornstetter@nt.com

\$Corresponding author: LIAFA (CNRS) - Université Paris 7 - 2, place Jussieu - 75251 Paris Cedex 05 - France - $e$-mail: dkeliafa.jussieu.fr

$\S$ IGM (CNRS) - Université de Marne la Vallée - 77454 Marne-la-Vallée Cedex - France - e-mail : jyt@univ-mlv.fr

II LIX (CNRS) - Ecole Polytechnique - 91128 Palaiseau Cedex - France -e-mail : katya@lix.polytechnique.fr 1365-8050 @ Maison de l’Informatique et des Mathématiques Discrètes (MIMD), Paris, France
} 
where the $u_{i}$ and $v_{i}$ 's denote independent centered complex Gaussian random variables with variances $E\left[\left|u_{i}\right|^{2}\right]=\chi_{i}$ and $E\left[\left|v_{i}\right|^{2}\right]=\delta_{i}$ (cf. Section 3.2).

The problem of evaluating such expressions was investigated by several researchers in signal processing (cf. $[1,4,10,12])$. The most interesting result in this direction is due to Barrett (cf. [1]) who obtained the expression

$$
P(U<V)=\sum_{k=1}^{N}\left(\prod_{i \neq k} \frac{1}{1-\delta_{k}^{-1} \delta_{i}} \prod_{i=1}^{N} \frac{1}{1+\delta_{k}^{-1} \chi_{i}}\right)
$$

referred to as Barrett's formula in the sequel.

Observe now that the expression (2) must be symmetric in the $\chi_{i}$ 's and in the $\delta_{i}$ 's due to its probabilistic interpretation given by (1). It is therefore natural to use the machinery of the theory of symmetric functions in order to analyze it more in depth. Using this point of view, we obtain several new expressions for Barrett's formula involving Schur functions, from which we derived an efficient and stable Bezoutian algorithm for computing in all cases the probability (1) (see also [3] for an elementary verification of the correctness of our algorithm). Note finally that the identities proved here are also connected with classical bijective combinatorics that can be used in order to deal with the numerous specializations of Barrett's formula involving degeneracies, i.e. the situations where some of the $\delta_{i}$ coincide (see [5] for more details).

\section{Symmetric function background}

We present here the background on symmetric functions which is used in our paper. More information about symmetric functions can be found in Macdonald's classical textbook [9]. We will somewhat deviate from the notation of [9] by considering partitions as weakly increasing sequences $I=\left(i_{1} \leq i_{2} \leq \ldots \leq i_{m}\right)$, instead as weakly decreasing ones. In the present context, this convention provides a more convenient presentation of the various matrices that will be encountered.

\subsection{Partitions}

Let $I$ be a partition, i.e. a finite nondecreasing sequence $I=\left(i_{1} \leq i_{2} \leq \ldots \leq i_{m}\right)$ of positive integers. The number $m$ of elements of $I$ is called the length of $I$. One can represent this partition by a Ferrers diagram of shape $I$, i.e. by a diagram of $i_{1}+\ldots+i_{m}$ boxes whose $i$-th row contains exactly $i_{k}$ boxes for each $1 \leq k \leq n$. The Ferrers diagram associated with the partition $I=(2,2,3)$ is for instance given below.

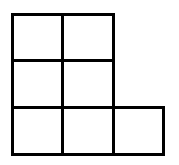

One says that a partition $I$ contains a partition $J$, which is denoted by $J \subset I$, if and only if the Ferrers diagram of $J$ is contained in the Ferrers diagram of $I$. The conjugate partition $I^{\sim}$ of $I$ is the partition obtained by reading the heights of the columns of the Ferrers diagram of $I$. One has for instance $I^{\sim}=$ $(1,3,3)$ when $I=(2,2,3)$ as can be seen in the previous picture.

When $I$ is a partition whose Ferrers diagram is contained in the square $\left(N^{N}\right)$ with $N$ rows of length $N$, one can also define the complementary partition $\bar{I}$ which is the conjugate of the partition $K$ whose Ferrers 
diagram is the complement (read from bottom to top) of the Ferrers diagram of $I$ in the square $\left(N^{N}\right)$. For instance, for $N=3$ and $I=(2,3)$, we have $K=(1,3)$ and $\bar{I}=(1,1,2)$ as can be checked on Figure 1 .

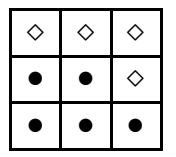

Fig. 1: Two complementary Ferrers diagrams.

\subsection{Symmetric functions}

Let $X$ be a set of indeterminates. The algebra of symmetric functions over $X$ is denoted by $\operatorname{Sym}(X)$. We define the complete symmetric functions $S_{k}(X)$ by their generating series

$$
\sigma_{z}(X)=\sum_{n=0}^{+\infty} S_{n}(X) z^{n}=\prod_{x \in X} \frac{1}{1-x z} .
$$

We also define in the same way the elementary symmetric functions $\Lambda_{k}(X)$ by their generating series (which is a polynomial when $X$ is finite)

$$
\lambda_{z}(X)=\sum_{n=0}^{+\infty} \Lambda_{n}(X) z^{n}=\prod_{x \in X}(1+x z)
$$

In order to use complete and elementary symmetric functions indexed by any integer $k \in \mathbb{Z}$, we also set $S_{k}(X)=\Lambda_{k}(X)=0$ for every $k<0$. Every symmetric function can be expressed in a unique way as a polynomial in the complete or elementary functions. For every $n$-tuple $I=\left(i_{1}, \ldots, i_{n}\right) \in \mathbb{Z}^{n}$, we now define the $S$ chur function $s_{I}(X)$ as the minor taken over the rows $1,2, \ldots, n$ and the columns $i_{1}+1, i_{2}+2, \ldots, i_{n}+n$ of the infinite matrix $\mathbb{S}=\left(S_{j-i}(X)\right)_{i, j \in \mathbb{Z}}$, i.e.

$$
s_{I}(X)=\left|\begin{array}{cccc}
S_{i_{1}}(X) & S_{i_{2}+1}(X) & \ldots & S_{i_{n}-n+1}(X) \\
S_{i_{1}-1}(X) & S_{i_{2}}(X) & \ldots & S_{i_{n}-n+2}(X) \\
\vdots & \vdots & \vdots & \vdots \\
S_{i_{1}-n+1}(X) & S_{i_{2}-n+2}(X) & \ldots & S_{i_{n}}(X)
\end{array}\right| .
$$

We also define more generally for every $I=\left(i_{1}, \ldots, i_{n}\right) \in \mathbb{Z}^{n}$ and $J=\left(j_{1}, \ldots, j_{n}\right) \in \mathbb{Z}^{n}$, the skew Schur function $s_{J / I}(X)$ as the minor of $\mathbb{S}$ taken over the rows $i_{1}+1, i_{2}+2, \ldots, i_{n}+n$ and the columns $j_{1}+1, j_{2}+$ $2, \ldots, j_{n}+n$. The importance of Schur functions comes from the fact that the family of the Schur functions indexed by partitions form a classical linear basis of the algebra of symmetric functions (see [9] for the details).

Let us finally introduce multi Schur functions (see [8]) which form another natural generalization of usual Schur functions that we will use in the sequel. Let $\left(X_{i}\right)_{1 \leq i \leq n}$ be a family of $n$ sets of indeterminates. Then, for every $n$-tuple $I=\left(i_{1}, \ldots, i_{n}\right) \in \mathbb{Z}^{n}$, one defines the multi Schur function $S_{I}\left(X_{1}, \ldots, X_{n}\right)$ by the 
determinantal formula

$$
s_{I}\left(X_{1}, \ldots, X_{N}\right)=\left|\begin{array}{cccc}
S_{i_{1}}\left(X_{1}\right) & S_{i_{2}+1}\left(X_{2}\right) & \ldots & S_{i_{n}-n+1}\left(X_{n}\right) \\
S_{i_{1}-1}\left(X_{1}\right) & S_{i_{2}}\left(X_{2}\right) & \ldots & S_{i_{n}-n+2}\left(X_{n}\right) \\
\vdots & \vdots & \vdots & \vdots \\
S_{i_{1}-n+1}\left(X_{1}\right) & S_{i_{2}-n+2}\left(X_{2}\right) & \ldots & S_{i_{n}}\left(X_{n}\right)
\end{array}\right| .
$$

Hence the usual Schur function $s_{I}(X)$ is the multi Schur function $s_{I}(X, \ldots, X)$.

\subsection{Transformations of alphabets}

Let $X$ and $Y$ be two sets of indeterminates. The complete symmetric functions of the formal set $X+Y$ are defined by their generating series

$$
\sigma_{z}(X+Y)=\sum_{n=0}^{\infty} S_{n}(X+Y) z^{n}=\sigma_{z}(X) \sigma_{z}(Y)
$$

One also defines the complete symmetric functions of the formal difference $X-Y$ by

$$
\sigma_{z}(X-Y)=\sum_{n=0}^{\infty} S_{n}(X-Y) z^{n}=\sigma_{z}(X) \lambda_{-z}(Y) .
$$

A symmetric function $F$ of the alphabet $X+Y$ or $X-Y$ is then an element of $\operatorname{Sym}(X) \otimes \operatorname{Sym}(Y)$ whose expression in this last algebra can be obtained by developing $F$ as a product of complete symmetric functions of $X+Y$ or $X-Y$ that are elements of $\operatorname{Sym}(X) \otimes \operatorname{Sym}(Y)$ according to the two defining relations (7) and (8). Note also that the complete symmetric functions of the formal set $-X$ are in particular defined by

$$
\sigma_{z}(-X)=\sum_{n=0}^{\infty} S_{n}(-X) z^{n}=\lambda_{-z}(X) .
$$

In other words, if $F(X)$ is a symmetric function of the set $X$, the symmetric function $F(-X)$ is obtained by applying to $F$ the algebra morphism that replaces $S_{n}(X)$ by $(-1)^{n} \Lambda_{n}(X)$ for every $n \geq 0$. Observe that the formal set $X-Y$ can also be defined by setting $X-Y=X+(-Y)$.

The expression of a Schur function of a formal sum of sets of indeterminates is in particular given by the Cauchy formula

$$
s_{I}(X+Y)=\sum_{J \subset I} s_{J}(X) s_{I / J}(Y)
$$

for every partition $\lambda$ (see [9]). One must also point out that one has

$$
s_{I / J}(-X)=(-1)^{|I|-|J|} S_{I^{\sim} / J}(X)
$$

for any partitions $\mu$ and $\lambda$ such that $\mu \subset \lambda$ (see again [9]). Note finally that the resultant of two polynomials can in particular be expressed as a rectangular Schur function of a difference of alphabets. Let $X$ and $Y$ be two sets of respectively $N$ and $M$ indeterminates. Then the expression

$$
R(X, Y)=\prod_{x \in X, y \in Y}(x-y)
$$

is the resultant of the polynomials that have $X$ and $Y$ as sets of roots and one can prove that one has $R(X, Y)=S_{N^{M}}(X-Y)($ see $[8,9])$. 


\subsection{Vertex operators}

We will use in the sequel the vertex operator $\Gamma_{z}(X)$ which transforms every symmetric function of $\operatorname{Sym}(X)$ into a series of $\operatorname{Sym}(X)\left[\left[z, z^{-1}\right]\right]$. Due to the fact that Schur functions indexed by partitions form a linear basis of $\operatorname{Sym}(X)$, we can therefore define this operator by setting

$$
\Gamma_{z}(X)\left(s_{I}(X)\right)=\sum_{m=-\infty}^{\infty} s_{(I, m)}(X) z^{m}
$$

for any partition $I=\left(I_{1}, \ldots, I_{n}\right)$, where we have here $(I, m)=\left(I_{1}, \ldots, I_{n}, m\right) \in \mathbb{Z}^{n+1}$ for every $m \in \mathbb{Z}$. The following formula (cf., e.g., [11] for a proof using the present notation) gives then another explicit expression of the action of a vertex operator on a Schur function.

Proposition 2.1 Let I be a partition. Then,

$$
\Gamma_{z}(X)\left(s_{I}(X)\right)=\sigma_{z}(X) s_{I}(X-1 / z) .
$$

\subsection{Lagrange's operators}

Let $X=\left\{x_{1}, \ldots, x_{N}\right\}$ be a finite alphabet of $N$ indeterminates. The Lagrange interpolating operator $L$ is the operator that maps every polynomial $f$ of $\mathbb{C}[X]$ which is symmetric in the last $N-1$ indeterminates, i.e. every element $f\left(x_{1}, X \backslash x_{1}\right)$ of $\operatorname{Sym}\left(x_{1}\right) \otimes \operatorname{Sym}\left(X \backslash x_{1}\right)$, to the symmetric polynomial $L(f)$ of $\operatorname{Sym}(X)$ defined by

$$
L(f)=\sum_{k=1}^{N} \frac{f\left(x_{k}, X \backslash x_{k}\right)}{R\left(x_{k}, X \backslash x_{k}\right)}
$$

where $R(A, B)$ stands again for the resultant of the two polynomials that have respectively the two sets of indeterminates $A$ and $B$ as sets of roots (cf Section 2.3). The following result, which is the algebraic expression of a geometrical property corresponding to the special case of Bott's formula for fibrations in projective lines (see $[6,7]$ for more details), gives an interesting property of the Lagrange interpolation operator.

Theorem 2.2 (Lascoux; [6]) Let $X=\left\{x_{1}, \ldots, x_{N}\right\}$ be an alphabet of $N$ indeterminates and let $\lambda=$ $\left(\lambda_{1}, \ldots, \lambda_{n}\right)$ be a partition that contains $\rho_{N-1}=(N-2, \ldots, 2,1,0)$. Then one has

$$
L\left(x_{1}^{k} s_{\lambda}\left(X \backslash x_{1}\right)\right)=s_{\lambda, k-N+1}(X)
$$

for every $k \geq 0$, where the Schur function involved in the right hand side of relation (13) is indexed by the sequence $(\lambda, k-N+1)=\left(\lambda_{1}, \ldots, \lambda_{n}, k-N+1\right)$ of $\mathbb{Z}^{n+1}$.

\section{Signal processing background}

\subsection{Demodulation with diversity}

Our initial motivation for studying Barrett's formula came from mobile communications. The probability $P(U<V)$ given by formula (1) appears naturally in the performance analysis of demodulation methods based on diversity which are standard in this context. 
Let us consider a model where one transmits an information $b \in\{-1,+1\}$ on a noisy channel. A reference $r$ (equal to 1 when emitted) is also sent on the same channel and at the same time as $b$. We assume that we receive $N$ pairs $\left(x_{i}(b), r_{i}\right)_{1 \leq i \leq N} \in(\mathbb{C} \times \mathbb{C})^{N}$ of data (the $x_{i}(b)$ 's) and references (the $r_{i}$ 's) $\|$ that have the following form

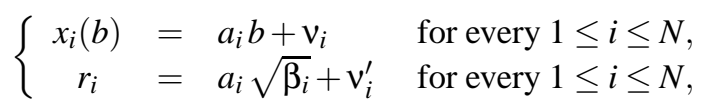

where $a_{i} \in \mathbb{C}$ is a complex number that models the channel fading associated with the transmission of the signals ${ }^{* *}$, where $\beta_{i} \in \mathbb{R}^{+}$is a positive real number that represents the excess of signal to noise ratio (SNR) available for the reference $r_{i}$ and where $v_{i}, v_{i}^{\prime} \in \mathbb{C}$ denote two independent complex white Gaussian noises. We also assume that every $a_{i}$ is a complex random variable distributed according to a Gaussian density of variance $\alpha_{i}$ for every $i \in[1, N]$.

According to these assumptions, all observables of our model, i.e. all pairs $\left(x_{i}(b), r_{i}\right)_{1 \leq i \leq N}$, are complex Gaussian random variables. We finally also assume that these $N$ observables are $N$ independent complex random variables. Under these hypotheses, one can then prove [2] that

$$
\log \left(\frac{P(b=+1 \mid X)}{P(b=-1 \mid X)}\right)=\sum_{i=1}^{N} \frac{4 \alpha_{i} \sqrt{\beta_{i}}}{1+\alpha_{i}\left(\beta_{i}+1\right)}\left(x_{i}(b) \mid r_{i}\right)
$$

with $X=\left(x_{i}(b), r_{i}\right)_{1 \leq i \leq N}$ and where $(\star \mid \star)$ denotes the Hermitian scalar product. The demodulation decision is based on the associated maximum likelihood criterium: one decides that $b$ was equal to 1 (resp. to $-1)$ when the right hand side of relation (14) is positive (resp. negative).

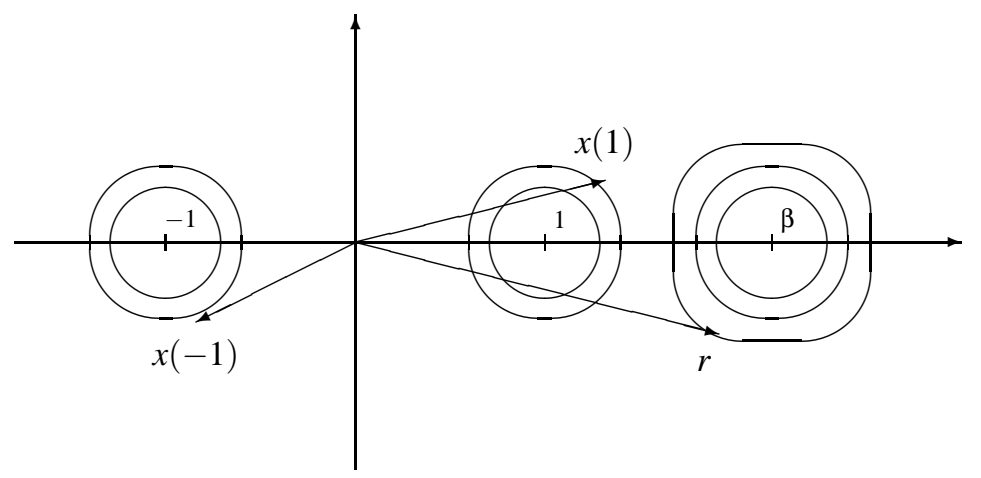

Fig. 2: Two possible noisy bits $x(1)$ and $x(-1)$ and a noisy reference $r$ in the case $N=1$.

Intuitively this means that one decides that the information $b=1$ was sent when the $x_{i}(b)$ 's are more or less globally in the same direction than the $r_{i}$ 's. Figure 2 illustrates the case $N=1$ and one can see that a

$\|$ This situation corresponds to spatial diversity, i.e. when more than one antenna is available for reception, which is typically the case in mobile communications.

** Fading is typically the result of the absorption of the signal by buildings. Its complex nature comes from the fact that it models both an attenuation (its modulus) and a phase shift (its argument). 
noisy reference $r$ has a positive (resp. negative) Hermitian scalar product with a noisy information $x$ when $x$ corresponds to a small perturbation of 1 (resp. -1 ).

The bit error probability (BER) of our model is the probability that the information $b=1$ was decoded as -1 , i.e. the probability that one had

$$
\sum_{i=1}^{N} \frac{4 \alpha_{i} \sqrt{\beta_{i}}}{1+\alpha_{i}\left(\beta_{i}+1\right)}\left(x_{i}(1) \mid r_{i}\right)<0
$$

Using the parallelogram identity, one can rewrite this last probability as

$$
P\left(\sum_{i=1}^{N}\left|u_{i}\right|^{2}-\sum_{j=1}^{N}\left|v_{i}\right|^{2}<0\right)
$$

where $u_{i}$ and $v_{i}$ denote the two variables defined by setting

$$
u_{i}=\left(\frac{\alpha_{i} \sqrt{\beta_{i}}}{1+\alpha_{i}\left(\beta_{i}+1\right)}\right)^{1 / 2}\left(x_{i}(1)+r_{i}\right) \quad \text { and } \quad v_{i}=\left(\frac{\alpha_{i} \sqrt{\beta_{i}}}{1+\alpha_{i}\left(\beta_{i}+1\right)}\right)^{1 / 2}\left(x_{i}(1)-r_{i}\right)
$$

for every $i \in[1, N]$. Our different hypotheses imply that the $u_{i}$ 's and the $v_{i}$ 's are independent complex Gaussian random variables. Hence the performance analysis of our model relies on the computation of a probability of error of type given by formula (1).

\subsection{Barrett's formula}

As we pointed out in Section 3.1, Barrett's formula is connected with performance analysis of demodulation methods based on diversity. More generally, the performance analysis of several other digital transmission systems relies on the computation of the probability that a Hermitian quadratic form $q$ in complex centered Gaussian variables is negative (cf. [10]). The problem of computing such a probability was adressed by several researchers of signal processing (see for instance [4, 12]). The most interesting result in this direction is due to Barrett (cf. [1]) who expressed the desired probability as a rational function of the eigenvalues of the covariance matrix associated with $q$. Formula (2) appears therefore as a special case of this general result.

For the sake of completeness, we will however show how to directly derive Barrett's formula (2). Let us consider the real random variables $U$ and $V$ defined by setting

$$
U=\sum_{i=1}^{N}\left|u_{i}\right|^{2} \quad \text { and } \quad V=\sum_{i=1}^{N}\left|v_{i}\right|^{2}
$$

where the $u_{i}$ 's and the $v_{i}$ 's are independent centered complex Gaussian random variables with variances equal to $E\left[\left|u_{i}\right|^{2}\right]=\chi_{i}$ and $E\left[\left|v_{i}\right|^{2}\right]=\delta_{i}$ for every $i \in[1, N]$. It is then easy to prove by induction on $N$ that the probability distribution functions of $U$ and $V$ are equal to

$$
d_{U}(x)=\sum_{j=1}^{N} \frac{\chi_{j}^{N-2}}{\prod_{1 \leq i \neq j \leq N}\left(\chi_{j}-\chi_{i}\right)} e^{-\frac{x}{\chi_{j}}} \text { and } \quad d_{V}(x)=\sum_{k=1}^{N} \frac{\delta_{k}^{N-2}}{\prod_{1 \leq i \neq k \leq N}\left(\delta_{k}-\delta_{i}\right)} e^{-\frac{x}{\delta_{k}}}
$$


when all variances $\chi_{i}$ and $\delta_{i}$ are distinct. Thus, one obtains

$$
P(V>x)=\int_{x}^{+\infty} d_{V}(t) d t=\sum_{k=1}^{N} \frac{\delta_{k}^{N-1}}{\prod_{1 \leq i \neq k \leq N}\left(\delta_{k}-\delta_{i}\right)} e^{-\frac{x}{\delta_{k}}}
$$

The probability of error (1) can now be expressed as

$$
P(U<V)=\int_{0}^{+\infty} d_{U}(x) P(V>x) d x .
$$

Using relations (15) and (16), this last identity leads us to the expression

$$
P(U<V)=\int_{0}^{+\infty} \sum_{j, k=1}^{N} \frac{\chi_{j}^{N-2} \delta_{k}^{N-1}}{\prod_{1 \leq i \neq j \leq N}\left(\chi_{j}-\chi_{i}\right) \prod_{1 \leq i \neq k \leq N}\left(\delta_{k}-\delta_{i}\right)} e^{-\frac{x}{\chi_{j}}} e^{-\frac{x}{\delta_{k}}} d x,
$$

from which we immediately get the relation

$$
P(U<V)=\sum_{j, k=1}^{N} \frac{\chi_{j}^{N-1} \delta_{k}^{N+1}}{\left(\delta_{k}+\chi_{j}\right) \prod_{1 \leq i \neq j \leq N}\left(\chi_{j}-\chi_{i}\right) \prod_{1 \leq i \neq k \leq N}\left(\delta_{k}-\delta_{i}\right)} .
$$

This last formula can now be rewritten as follows

$$
P(U<V)=\sum_{k=1}^{N} \frac{\delta_{k}^{N+1}}{\prod_{i=1}^{N}\left(\delta_{k}+\chi_{i}\right) \prod_{1 \leq i \neq k \leq N}\left(\delta_{k}-\delta_{i}\right)}\left(\sum_{j=1}^{N} \frac{\prod_{1 \leq i \neq k \leq N}\left(\delta_{k}+\chi_{i}\right)}{\prod_{1 \leq i \neq k \leq N}\left(\chi_{j}-\chi_{i}\right)} \chi_{j}^{N-1}\right)
$$

from which we can deduce the formula

$$
P(U<V)=\sum_{k=1}^{N} \frac{\delta_{k}^{2 N-1}}{\prod_{i=1}^{N}\left(\delta_{k}+\chi_{i}\right) \prod_{\substack{1 \leq i \leq N \\ i \neq k}}\left(\delta_{k}-\delta_{i}\right)}
$$

due to the fact that the inner sum in relation (17) is just the Lagrange interpolation expression taken at the points $\left(-\chi_{j}\right)_{1 \leq j \leq N}$ of the polynomial $\delta_{k}^{N-1}$ (considered here as a polynomial of $\mathbb{C}\left[\chi_{1}, \ldots, \chi_{N}\right]\left[\delta_{k}\right]$ ). Formula (2) follows then immediately from this last expression of the probability of error that we are studying.

\section{Symmetric functions expressions for Barrett's formula}

This section is devoted to a formulation of Barrett's formula (18) in terms of symmetric functions. We will therefore use here the notations of Section 3.2. 


\subsection{A combinatorial formula}

Note first that Barrett's formula can be easily expressed using the Lagrange operator $L$ introduced in Section 2.5. Indeed, let us set $\delta_{k}=x_{k}$ and $\chi_{k}=-y_{k}$ for every $k \in[1, N]$. Then one can rewrite formula (18) in the following way:

$$
P(U<V)=\sum_{k=1}^{N} \frac{x_{k}^{2 N-1}}{R\left(x_{k}, Y\right) R\left(x_{k}, X \backslash x_{k}\right)}
$$

where we denoted $X=\left\{x_{1}, \ldots, x_{N}\right\}$ and $Y=\left\{y_{1}, \ldots, y_{N}\right\}$ and where $R(A, B)$ stands for the resultant of two polynomials as defined in Section 2.3. Hence we have

$$
P(U<V)=\sum_{k=1}^{N} \frac{g\left(x_{k}, X \backslash x_{k}\right)}{R\left(x_{k}, X \backslash x_{k}\right)}=L(g)
$$

where $g$ stands for the element of $\operatorname{Sym}\left(x_{1}\right) \otimes \operatorname{Sym}\left(X \backslash x_{1}\right)$ defined by

$$
g\left(x_{1}, X \backslash x_{1}\right)=g\left(x_{1}\right)=\frac{x_{1}^{2 N-1}}{R\left(x_{1}, Y\right)} .
$$

Observe now that one has

$$
g\left(x_{1}, X \backslash x_{1}\right)=\frac{1}{R(X, Y)} x_{1}^{2 N-1} f\left(x_{1}, X \backslash x_{1}\right)
$$

where $f$ stands for the element of $\operatorname{Sym}\left(x_{1}\right) \otimes \operatorname{Sym}\left(X \backslash x_{1}\right)$ defined by

$$
f\left(x_{1}, X \backslash x_{1}\right)=R\left(X \backslash x_{1}, Y\right)=s_{\left(N^{N-1}\right)}\left(\left(X \backslash x_{1}\right)-Y\right)
$$

(the last above equality comes from the expression of the resultant in terms of Schur functions given in Section 2.3). Note now that the resultant $R(X, Y)$, being symmetric in the alphabet $X$, is a scalar for the operator $L$. It follows therefore from relation (19) that one has

$$
P(U<V)=\frac{L\left(x_{1}^{2 N-1} f\left(x_{1}, X \backslash x_{1}\right)\right)}{R(X, Y)} .
$$

Let us now study the numerator of the right-hand side of relation (21) to get another expression for $P(U<V)$. Note first that the Cauchy formula yields the expansion

$$
s_{\left(N^{N-1}\right)}\left(\left(X \backslash x_{1}\right)-Y\right)=\sum_{I \subset\left(N^{N-1}\right)} s_{I}\left(X \backslash x_{1}\right) s_{\left(N^{N-1}\right) / I}(-Y) .
$$

According to the identities (20) and (22), we now get the relations

$$
\begin{aligned}
L\left(x_{1}^{2 N-1} f\left(x_{1}, X \backslash x_{1}\right)\right) & =\sum_{I \subset\left(N^{N-1}\right)} L\left(x_{1}^{2 N-1} s_{I}\left(X \backslash x_{1}\right)\right) s_{\left(N^{N-1}\right) / I}(-Y) \\
& =\sum_{I \subset\left(N^{N-1}\right)} s_{(I, N)}(X) s_{\left(N^{N-1}\right) / I}(-Y),
\end{aligned}
$$


the last above equality being an immediate consequence of Theorem 2.2. Using the expression given by relation (11) for $s_{\left(N^{N-1}\right) / I}(-Y)$ and going back to the definition of a skew Schur function given in Section 2.2 , we can rewrite the last above expression as

$$
L\left(x_{1}^{2 N-1} f\left(x_{1}, X \backslash x_{1}\right)\right)=\sum_{I \subset\left(N^{N-1}\right)}(-1)^{|I|} s_{(I, N)}(X) s_{\overline{(I, N)}}(Y)
$$

where $\overline{(I, N)}$ denotes the complementary partition of $(I, N)$ in the square $N^{N}$ (cf. Section 2.1). Going back to the initial variables, the signs disappear in the previous formula by homogeneity of Schur functions. Substituting the obtained identity in (21), we finally get an expression in terms of Schur functions for the probability $P(U<V)$, namely

$$
P(U<V)=\frac{\sum_{I \subset\left(N^{N-1}\right)} s_{(I, N)}(\chi) s_{\overline{(I, N)}}(\Delta)}{\prod_{1 \leq i, j \leq N}\left(\chi_{i}+\delta_{j}\right)}
$$

where we set $\chi=\left\{\chi_{1}, \ldots, \chi_{N}\right\}$ and $\Delta=\left\{\delta_{1}, \ldots, \delta_{N}\right\}$.

Example 4.1 For $N=2$, one can indeed check that Barrett's formula (2) reduces to

$$
P(U<V)=\frac{\chi_{1} \chi_{2}\left(\delta_{1}^{2}+\delta_{1} \delta_{2}+\delta_{2}^{2}\right)+\left(\chi_{1}+\chi_{2}\right)\left(\delta_{1}^{2} \delta_{2}+\delta_{1} \delta_{2}^{2}\right)+\delta_{1}^{2} \delta_{2}^{2}}{\left(\chi_{1}+\delta_{1}\right)\left(\chi_{1}+\delta_{2}\right)\left(\chi_{2}+\delta_{1}\right)\left(\chi_{2}+\delta_{2}\right)}
$$

which is equal (as expected) to

$$
P(U<V)=\frac{s_{1,1}\left(\chi_{1}, \chi_{2}\right) s_{2}\left(\delta_{1}, \delta_{2}\right)+s_{1}\left(\chi_{1}, \chi_{2}\right) s_{1,2}\left(\delta_{1}, \delta_{2}\right)+s_{2,2}\left(\delta_{1}, \delta_{2}\right)}{\prod_{1 \leq i, j \leq 2}\left(\chi_{i}+\delta_{j}\right)} .
$$

\subsection{A determinantal formula}

Let us work again with the alphabets $X$ and $Y$ defined in Section 4.1. We saw there that

$$
P(U<V)=\frac{f_{N}(X, Y)}{R(X, Y)}
$$

where $f_{N}(X, Y)$ is the symmetric function of $\operatorname{Sym}(X) \otimes \operatorname{Sym}(Y)$ given by

$$
f_{N}(X, Y)=\sum_{I \subset\left(N^{N-1}\right)} s_{(I, N)}(X) s_{\left(N^{N-1}\right) / I}(-Y) .
$$

Let us now compute the action of the vertex operator $\Gamma_{z}(X)$ on the rectangular usual Schur function $s_{\left(N^{N-1}\right)}(X-Y)$. Note first that the Cauchy formula shows again that

$$
s_{\left(N^{N-1}\right)}(X-Y)=\sum_{I \subset\left(N^{N-1}\right)} s_{I}(X) s_{\left(N^{N-1}\right) / I}(-Y) .
$$


Applying the vertex operator $\Gamma_{z}(X)$ on this expansion, we now get

$$
\begin{aligned}
\Gamma_{z}(X)\left(s_{\left(N^{N-1}\right)}(X-Y)\right) & =\sum_{I \subset\left(N^{N-1}\right)} \Gamma_{z}(X)\left(s_{I}(X)\right) s_{\left(N^{N-1}\right) / I}(-Y) \\
& =\sum_{m=-\infty}^{+\infty}\left(s_{(I, m)}(X) s_{\left(N^{N-1}\right) / I}(-Y)\right) z^{m}
\end{aligned}
$$

Hence $f_{N}(X, Y)$ is equal to the coefficient of $z^{N}$ in the image of $s_{\left(N^{N-1}\right)}(X-Y)$ by $\Gamma_{z}(X)$. On the other hand, using the Cauchy formula in connection with relation (12), one can also write

$$
\begin{aligned}
\Gamma_{z}(X)\left(s_{\left(N^{N-1}\right)}(X-Y)\right) & =\sigma_{z}(X) s_{\left(N^{N-1}\right)}(X-Y-1 / z) \\
& =\sigma_{z}(X)\left(\sum_{j=0}^{+\infty} s_{\left(N^{N-1}\right) /\left(1^{j}\right)}(X-Y) s_{\left(1^{j}\right)}(-1 / z)\right) \\
& =\left(\sum_{i=0}^{\infty} s_{i}(X) z^{i}\right)\left(\sum_{j=0}^{\infty} s_{\left(N^{N-1}\right) /\left(1^{j}\right)}(X-Y)(-1 / z)^{j}\right)
\end{aligned}
$$

due to the fact that the only non zero Schur functions of the alphabet $-1 / z$ are indexed by column partitions of the form $1^{k}$ (and are equal to $(-1 / z)^{k}$ ). The coefficient of $z^{N}$ in the above product gives us then a new expression for $f_{N}(X, Y)$, namely

$$
f_{N}(X, Y)=\sum_{k=0}^{N-1}(-1)^{k} s_{N+k}(X) s_{N^{N-1} / 1^{k}}(X-Y)
$$

But this last expression is just the expansion along the last colomn of the determinant

$$
\left|\begin{array}{ccccc}
s_{N}(X-Y) & s_{N+1}(X-Y) & \ldots & s_{2 N-2}(X-Y) & s_{2 N-1}(X) \\
s_{N-1}(X-Y) & s_{N}(X-Y) & \ldots & s_{2 N-3}(X-Y) & s_{2 N-3}(X) \\
\vdots & \vdots & \ddots & \vdots & \vdots \\
s_{1}(X-Y) & s_{2}(X-Y) & \vdots & s_{N-1}(X-Y) & s_{N}(X)
\end{array}\right|
$$

which is the determinantal expression of the multi Schur fonction $s_{\left(N^{N}\right)}(X-Y, \ldots, X-Y, X)$ (see Section 2.3). Hence relation (26) gives us both a determinantal and a multi Schur function expression for the denominator of the right hand side of formula (24). Using the interpretation of the resultant $R(X, Y)$ as a multi Schur function (see Section 2.3), we now get that

$$
P(U<V)=\frac{s_{\left(N^{N}\right)}(X-Y, \ldots, X-Y, X)}{s_{\left(N^{N}\right)}(X-Y, \ldots, X-Y)}
$$

where the alphabet $X-Y$ appears $N-1$ times in the denominator and $N$ times in the numerator of the right hand side of the above formula. 


\section{An efficient and stable algorithm for the error probability}

\subsection{A Toeplitz system and its solution}

Using the determinantal expression of the multi Schur function $s_{\left(N^{N}\right)}(X-Y, \ldots, X-Y)$, we can now observe that relation (27) shows that the probability $P(U<V)$ is equal to the quotient of the determinant (26) by the determinant

$$
\left|\begin{array}{cccc}
s_{N}(X-Y) & s_{N+1}(X-Y) & \ldots & s_{2 N-1}(X-Y) \\
s_{N-1}(X-Y) & s_{N}(X-Y) & \ldots & s_{2 N-2}(X-Y) \\
\vdots & \vdots & \vdots & \vdots \\
s_{1}(X-Y) & s_{2}(X-Y) & \vdots & s_{N}(X-Y)
\end{array}\right|
$$

where the former determinant is obtained by replacing the last column of the determinant (26) by the $N$-dimensional vector $\left(s_{2 N-1}(X), s_{2 N-2}(X), \ldots, s_{N}(X)\right)$. Hence the right hand side of relation (27) can be interpreted as the Cramer expression for the last component $p_{0}$ of the linear system

$$
\left(\begin{array}{cccc}
s_{N}(X-Y) & s_{N+1}(X-Y) & \cdots & s_{2 N-1}(X-Y) \\
s_{N-1}(X-Y) & s_{N}(X-Y) & \cdots & s_{2 N-2}(X-Y) \\
\vdots & \vdots & \ddots & \vdots \\
s_{1}(X-Y) & s_{2}(X-Y) & \cdots & s_{N}(X-Y)
\end{array}\right)\left(\begin{array}{c}
p_{N-1} \\
p_{N-2} \\
\vdots \\
p_{0}
\end{array}\right)=\left(\begin{array}{c}
s_{2 N-1}(X) \\
s_{2 N-2}(X) \\
\vdots \\
s_{N}(X)
\end{array}\right) .
$$

Let us now set $\pi(t)=p_{0}+p_{1} t+\cdots+p_{N-1} t^{N-1}$. This last linear system expresses that the coefficients of order $N, N+1, \ldots, 2 N-1$ in the series $\pi(t) \sigma_{t}(X-Y)$ are equal to the coefficients of the same order in $\sigma_{t}(X)$. This means equivalently that there exists a polynomial $\mu(t)$ of degree less or equal to $N-1$ such that one has

$$
\pi(t) \sigma_{t}(X-Y)-\sigma_{t}(X)+\mu(t)=O\left(t^{2 N}\right)
$$

Going back to the definition of $\sigma_{t}(X-Y)$, we see that this last property can be rewritten as

$$
\left(\pi(t) \lambda_{-t}(Y)-1\right) \sigma_{t}(X)+\mu(t)=O\left(t^{2 N}\right)
$$

which is itself clearly equivalent to the fact that

$$
\pi(t) \lambda_{-t}(Y)+\mu(t) \lambda_{-t}(X)=1+O\left(t^{2 N}\right)
$$

Since the left hand side of the above identity is a polynomial of degree at most $2 N-1$, it follows that its right hand side must be equal to 1 . Hence we have shown that one has

$$
\pi(t) \lambda_{-t}(Y)+\mu(t) \lambda_{-t}(X)=1
$$

It follows that the probability $P(U<V)$ is the constant term $\pi(0)$ of the Bezout polynomial $\pi(t)$ defined by (28). 


\subsection{A Bezoutian algorithm}

It is now immediate to see that the results of Section 5.1 imply that the following algorithm computes the error probability $P(U<V)$.

- Step 1. Consider the two polynomials $X(z)$ and $\Delta(z)$ of $\mathbb{R}[z]$ defined by setting

$$
X(z)=\prod_{i=1}^{N}\left(1-\chi_{i} z\right) \quad \text { and } \quad \Delta(z)=\prod_{i=1}^{N}\left(1+\delta_{i} z\right) .
$$

- Step 2. Compute the unique polynomial $\pi(z)$ of $\mathbb{R}[z]$ of degree $d(\pi) \leq N-1$ such that

$$
\pi(z) X(z)+\mu(z) \Delta(z)=1
$$

where $\mu(z)$ stands for some polynomial of $\mathbb{R}[z]$ of degree $d(\mu) \leq N-1$.

- Step 3. Evaluate $\pi(0)=P(U<V)$.

Due to the fact the above second step can be realized by the classical generalized Euclidean algorithm, our algorithm has the same complexity (i.e. quadratic in $N$ ) as Barrett's formula and is hence algorithmically rather efficient. On the other hand, our algorithm is also numerically stable (which was not the case with Barrett's formula (2)) since it inherits this property from the numerical stability of the generalized Euclidean algorithm. Note finally that one can also directly derive our algorithm from elementary techniques (cf. [3]) which does however not give any insight in the internal structure of Barrett's formula.

\section{Acknowledgements}

The authors would like to thank one of the anonymous referees for his/her (?) numerous vuluable remarks and his/her (?) careful reading of the paper.

\section{References}

[1] BARRETT M., Error probability for optimal and suboptimal quadratic receivers in rapid Rayleigh fading channels, IEEE Trans. Select. Areas in Commun., 302-304, February 1987.

[2] Dornstetter J.L., Personal communication.

[3] Dornstetter J.L., Krob D., Thibon J.Y., Fast and Stable Computation of Error Probability in Rapid Rayleigh Fading Channels, LIAFA Technical Report, Paris, 2000, submitted.

[4] Iмноғ J.P., Computing the distribution of quadratic forms in normal variables, Biometrika, 48, 419-426, 1961.

[5] Krob D., VASSilieva E.A., Performance analysis of modulation with diversity - A combinatorial approach II: Bijective methods, LIAFA Technical Report, Paris, 2001.

[6] Lascoux A., Inversion des matrices de Hankel, Lin. Alg. and its Appl., 129, 77-102, 1990 
[7] Lascoux A., SchütZenberger M.P., Symmetry ang Flag Manifolds, Lect. Notes in Maths., 996, 118-144, Springer Verlag, 1982

[8] Lascoux A., Schützenberger M.P., Formulaire raisonné de fonctions symétriques, Public. LITP, Paris 7, 1985.

[9] Macdonald I.G., Symmetric Functions and Hall Polynomials, Oxford, 1979.

[10] Proakis J., Digital Communications, 3rd Edition, McGraw-Hill, 1995.

[11] ThiвON J.Y., Hopf algebras of symmetric functions and tensor products of symmetric group representations, Int. J. of Alg. and Comput., 1, (2), 207-221, 1991.

[12] TURIN G.L., The characteristic function of Hermitian quadratic forms in complex normal variables, Biometrika, 47, 199-201, 1960. 